This work is a copy of the published version:

M. Schmidt, T. Pfeiffer, C. Grill, R. Huber, and C. Jirauschek, "Coexistence of Intensity Pattern Types in Broadband Fourier Domain Mode Locked (FDML) Lasers," Proceedings of the 2019 Conference on Lasers and ElectroOptics Europe \& European Quantum Electronics Conference (CLEO ${ }^{\circledR} /$ Europe-EQEC), 23-27 June, 2019, Munich, Germany, paper ef_p_5, doi: 10.1109/CLEOE-EQEC.2019.8872381

(C2019 IEEE. Personal use of this material is permitted. Permission from IEEE must be obtained for all other uses, in any current or future media, including reprinting/republishing this material for advertising or promotional purposes, creating new collective works, for resale or redistribution to servers or lists, or reuse of any copyrighted component of this work in other works. 


\title{
Coexistence of Intensity Pattern Types in Broadband Fourier Domain Mode Locked (FDML) Lasers
}

\author{
Mark Schmidt $^{1}$, Tom Pfeiffer ${ }^{2}$, Christin Grill ${ }^{2}$, Robert Huber $^{2}$, Christian Jirauschek $^{1}$ \\ 1. Department of Electrical and Computer Engineering, Technical University of Munich, Arcisstraße 21, 80333 Munich, Germany \\ 2. Institut für Biomedizinische Optik, Universität zu Lübeck, Peter-Monnik-Weg 4, 23562 Lübeck, Germany
}

Fourier domain mode locked (FDML) lasers, in which the sweep period of the swept bandpass filter is synchronized with the roundtrip time of the optical field, are broadband and rapidly tunable fiber ring laser systems, which offer rich dynamics. A detailed understanding is important from a fundamental point of view, and also required in order to improve current FDML lasers which have not reached their coherence limit yet. Here, we study the formation of localized patterns in the intensity trace of FDML laser systems based on a master equation approach [1] derived from the nonlinear Schrödinger equation for polarization maintaining setups, which shows excellent agreement with experimental data. A variety of localized patterns and chaotic or bistable operation modes were previously discovered in [2-4] by investigating primarily quasi-static regimes within a narrow sweep bandwidth where a delay differential equation model was used. In particular, the formation of so-called holes which are characterized by a dip in the intensity trace and a rapid phase jump are described. Such holes have tentatively been associated with Nozaki-Bekki holes which are solutions to the complex Ginzburg-Landau equation. In Fig. 1 (b) to (d) small sections of a numerical solution of our master equation are presented for a partially dispersion compensated polarization maintaining FDML laser setup. Within our approach, we are able to study the full sweep dynamics over a broad sweep range of more than $100 \mathrm{~nm}$. This allows us to identify different co-existing intensity patterns within a single sweep. In general, high frequency distortions in the intensity trace of FDML lasers [5] are mainly caused by synchronization mismatches caused by the fiber dispersion or a detuning of the roundtrip time of the optical field to the sweep period of the swept bandpass filter. This timing errors lead to rich and complex dynamics over many roundtrips and are a major source of noise, greatly affecting imaging and sensing applications. For example, the imaging quality in optical coherence tomography where FDML lasers are superior sources is significantly reduced [5].
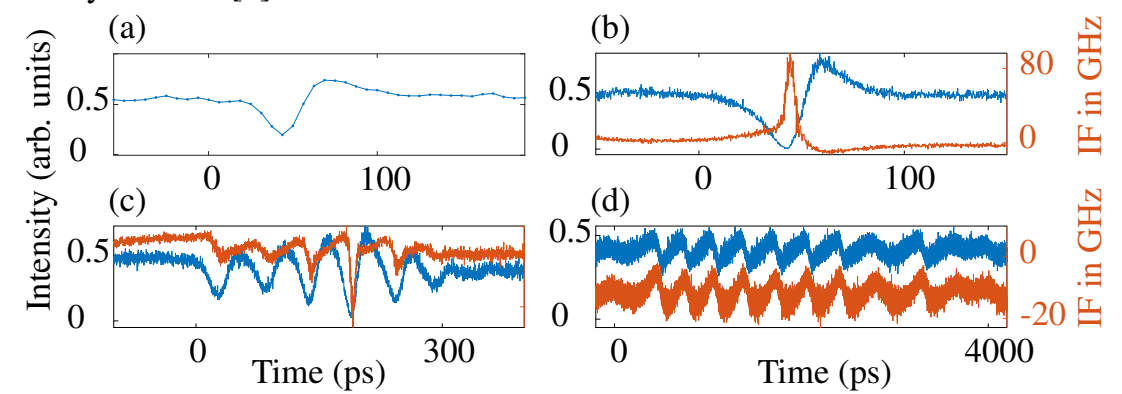

Fig. 1 (a) Measured hole in the intensity trace of a partially dispersion compensated laser with a sweep bandwidth of $117 \mathrm{~nm}$ around $1292 \mathrm{~nm}$ and a sweep rate of $3.2 \mathrm{MHz}$, recorded with $50 \mathrm{GHz}$ analog bandwidth. (b) Simulated hole in a similar setup as in (a). (c) Fringe-like instabilities in the simulated trace. (d) Periodic pattern near the turning point of forward and backward sweep which was also reported in [2] and, is referred as Turing-type instability therein in the context of a detuned laser.

The most often observed localized solutions are summarized in Fig. 1. In order to investigate the rapid phase jumps associated with the hole solution, we extract the instantaneous frequency (IF) of the instantaneous optical phase. The dips in the intensity trace are caused by a dynamic long term effect which is a result of the interplay between the swept bandpass filter, the semiconductor optical amplifier (SOA) gain medium and the optical fiber. The fiber nonlinearity plays a negligible role in this process whereas the nonlinearity in the SOA gain medium dominantly influences the shape of the pattern, in addition to the fiber dispersion and the filter's time domain response. We believe that our master equation approach is perfectly suitable to contribute to a further understanding and control of noise in FDML lasers and is a perfect starting point for systematically deriving analytical solutions which are able to prove the existence of Nozaki-Bekki holes.

\section{References}

[1] C. Jirauschek and R. Huber, "Efficient simulation of the swept-waveform polarization dynamics in fiber spools and Fourier domain modelocked (FDML) lasers," J. Opt. Soc. Am. B 34, 1135-1146 (2017).

[2] S. Slepneva, B. Kelleher, B. O'Shaughnessy, S. P. Hegarty, A. G. Vladimirov, and G. Huyet, "Dynamics of Fourier domain mode-locked lasers," Opt. Express 21, 19240—19251 (2013).

[3] A. Pimenov, S. Slepneva, G. Huyet, and A. G. Vladimirov, "Dispersive time-delay dynamical systems,” Phys. Rev. Lett. 118, 193901 (2017).

[4] S. Slepneva, B. O'Shaughnessy, A. Vladimirov, S. Rica, and G. Huyet, “Turbulent laser puffs,” arXiv preprint arXiv:1707.08304 (2017).

[5] T. Pfeiffer, M. Petermann, W. Draxinger, C. Jirauschek, and R. Huber, "Ultra low noise Fourier domain mode locked laser for high quality megahertz optical coherence tomography," Biomed. Opt. Express 9, 4130-4148 (2018). 